\title{
Pre, intra and post-ischemic hypothermic neuroprotection in temporary focal cerebral ischemia in rats: morphometric analysis
}

\author{
Neuroproteção hipotérmica pré, intra e pós-isquêmica na isquemia cerebral focal \\ temporária em ratos: análise morfométrica
}

Roberto Alexandre Dezena ${ }^{1}$, Benedicto Oscar Colli², Carlos Gilberto Carlotti Junior ${ }^{3}$, Luís Fernando Tirapelli4

\begin{abstract}
Objective: To evaluate the neuroprotection of mild hypothermia, applied in different moments, in temporary focal cerebral ischemia in rats. Methods: Rats was divided into Control (C), Sham (S), Ischemic-control(IC), Pre-ischemic Hypothermia (IH1), Intra-ischemic Hypothermia $(\mathrm{IH} 2)$, and Post-ischemic Hypothermia (IH3) groups. Morphometry was performed using the KS400 software (Carl Zeiss $\left.{ }^{\circledR}\right)$ in coronal sections stained by Luxol Fast Blue. Ischemic areas and volumes were obtained. Results: Statistically, blue areas showed difference for C vs. IC, IC vs. $I H 1$ and IC vs. IH2 ( $p=0.0001 ; p=0.01 ; p=0.03)$, and no difference between C vs. S, IC vs. IH3 and IH vs. IH2 ( $p=0.39 ; p=0.85 ; p=0.63)$. Red areas showed difference between C vs. IC, IC vs. IH1 and IC vs. IH2 ( $p=0.0001 ; p=0.009 ; p=0.03$ ), and no difference between C vs. S, IC vs. IH3 and IH1 vs. IH 2 ( $p=0.48 ; p=0.27 ; p=0.68)$. Average ischemic areas and ischemic volumes showed difference between IC vs. IH1 and IC vs. IH2 ( $p=0.0001$ and $p=0.0011$ ), and no difference between IC vs. IH3 and IH1 vs. IH2 ( $p=0.57 ; p=0.79)$. Conclusion: Pre-ischemic and intra-ischemic hypothermia were shown to be similarly neuroprotective, but this was not true for post-ischemic hypothermia.
\end{abstract}

Key words: brain ischemia, mild hypothermia, morphometry, neuroprotection, reperfusion.

RESUMO

Objetivo: Avaliar a neuroproteção da hipotermia leve, aplicada em diferentes momentos, durante isquemia cerebral focal temporária em ratos .Métodos: Ratos foram divididos em grupos: Controle (C), Sham (S), Controle-isquêmico (IC), Hipotermia Pré-isquêmica (IH1), Hipotermia Intra-isquêmica (IH2) e Hipotermia Pós-isquêmica (IH3). A morfometria foi realizada em secções coronais coradas por Luxol Fast Blue através do programa KS400 (Carl Zeiss $\left.{ }^{\circledR}\right)$. Foram calculados áreas e volumes isquêmicos. Resultados: Estatisticamente, áreas azuis demonstraram diferença entre os grupos $\mathrm{C}$ vs. IC, IC vs. IH1 e IC vs. IH2 ( $p=0,0001 ; p=0,01 ; p=0,03$ ), e nenhuma diferença entre $C$ vs. $S$, IC vs. $I H 3$ e IH vs. IH2 ( $p=0,39 ; p=0,85 ; p=0,63)$. Áreas vermelhas demonstraram diferença entre $C$ vs. IC, IC vs. $I H 1$ e IC vs. $I H 2(p=0,0001 ; p=0,009$; $p=0,03$ ), e nenhuma diferença entre $C$ vs. $S$, IC vs. IH3 e IH1 vs. IH2 ( $p=0,48 ; p=0,27 ; p=0,68)$. Áreas isquêmicas médias e volumes isquêmicos demonstraram diferença entre os grupos IC vs. IH1 e IC vs. IH2 ( $p=0,0001$ and p=0,0011), e nenhuma diferença entre IC vs. IH3 and IH1 vs. IH2 ( $p=0,57 ; p=0,79)$. Conclusão: Hipotermias pré-isquêmica e intra-isquêmica demonstraram neuroproteção em grau semelhante, o que não ocorreu com hipotermia pós-isquêmica.

Palavras-Chave: isquemia encefálica, hipotermia leve, morfometria, neuroproteção, reperfusão.

Experimental Neurosurgery Laboratory, Department of Surgery and Anatomy, Ribeirão Preto Medical School, Universidade de São Paulo SP, Brazil. 'M.D., Ph.D.; Neurosurgeon, Division of Neurosurgery, Department of Surgery and Anatomy, Ribeirão Preto Medical School, Universidade de São Paulo (USP), Ribeirão Preto SP, Brazil; Division of Neurosurgery, Universidade Federal doTriângulo Mineiro (UFTM), Uberaba MG, Brazil;

${ }^{2}$ M.D., Ph.D.; Professor of Neurosurgery, Head and Chairman, Division of Neurosurgery, Department of Surgery and Anatomy, Ribeirão Preto Medical School, USP, São Paulo SP, Brazil;

${ }^{3}$ M.D., Ph.D.; Professor of Neurosurgery, Division of Neurosurgery, Department of Surgery and Anatomy, Ribeirão Preto Medical School, USP, São Paulo SP, Brazil; 4 M.Sc., Ph.D.; Professor of Anatomy, Division of Anatomy, Department of Surgery and Anatomy, Ribeirão Preto Medical School, USP, São Paulo SP, Brazil. Correspondence: Roberto Alexandre Dezena; Rua Veríssimo 1.264/202-2; 38022-090 Uberaba MG - Brasil; E-mail: rdezena@yahoo.com.br Support: Ribeirão Preto Medical School, Universidade de São Paulo (FMRP-USP); Fundação de Amparo à Pesquisa do Estado de São Paulo (FAPESP); Conselho Nacional de Desenvolvimento Científico e Tecnológico (CNPq).

Conflict of interest: There is no conflict of interest to declare.

Received 04 March 2012; Received in final form 11 April 2012; Accepted 18 April 2012 
Cerebral ischemia is a very common disease, with unpredictable clinical outcome, and limited prophylaxis and treatment. Vasospasm after subarachnoid hemorrhage and temporary vascular occlusion on vascular microneurosurgery are the main situations of focal cerebral ischemia for the neurosurgeon. Marked decrease of cerebral blood flow triggers a series of biochemical events, with histopathological consequences which, if not blocked, leads to neuronal death. Three concentric areas are histopathologically defined in focal ischemia: the central ischemic zone, the ischemic penumbra zone and the external zone of normal tissue ${ }^{1}$. In ischemic penumbra zone initially the cells remain morphologically normal, but lose their electrical function, featuring an isoelectric electroencephalogram and, depending on the time of ischemia and on the anastomotic supply, cell death will occur. Moreover, this area is the most frequent site of apoptosis ${ }^{1}$. On the other hand, this area is likely to save when neuroprotective strategies are adopted. Several animals have been used for experimental studies on cerebral ischemia, and among them, the rat is the most used ${ }^{2}$. The neuroprotective effect of hypothermia in experimental cerebral ischemia is described since 1950 's $\mathrm{s}^{3}$. Neuroprotection afforded by hypothermia is classically attributed to reduction of metabolic demands. In addition, other mechanisms have been proposed $^{1,4,5}$. Hypothermia can be applied at different times of ischemia: before (pre-ischemic), during (intra-ischemic), and after ischemia (post-ischemic). The intra-ischemic hypothermia is the most studied, and it is the most effective ${ }^{4}$. This modality of hypothermia significantly reduces the total volume of experimental infarction in the basal ganglia and $\operatorname{cortex}^{6,7}$, and also improves clinical evaluation ${ }^{8}$. In the post-ischemic mode, hypothermia acts mainly by inhibiting the "reperfusion injury". Although less effective than intra-ischemic hypothermia, it reduces experimental neuronal loss in hippocampus induced by ischemia $^{10}$, reduce the volume of cerebral infarction ${ }^{6,11}$ and typically reduces cortical infarct volume ${ }^{12}$. Classically, to obtain the maximum neuroprotective effect with this mode of hypothermia in experimental temporary ischemia, it must be instituted within 15 minutes after reperfusion ${ }^{13}$. However, recent studies demonstrate a viable window of tolerance up three to four hours to get maximum of neuroprotection ${ }^{14,15}$. This window can vary according the grade and duration of hypothermia ${ }^{4}$. As far as our knowledge, there are no reports in the literature about the effect of pre-ischemic hypothermia. This study aimed to compare the neuroprotective effect of mild hypothermia (32$34^{\circ} \mathrm{C}$ ) at different times of ischemia: (pre, intra and post-ischemia) in rats submitted to focal cerebral ischemia by temporary occlusion of the middle cerebral artery (MCA).

\section{METHODS}

This study was approved by the Ethics Committee on Animal Research, Ribeirão Preto Medical School, Universidade de São Paulo (Protocol n¹08/2009).The experiments were performed according to the standards proposed by the Brazilian College of Animal Experimentation and the International Council for Laboratory Animal Science. We used adult male Wistar rats, weighing 280-310 g. Before the surgical procedure, the animals were randomly distributed, by ballot, in six groups. All groups, except the Control and Sham were submitted to 60 minutes of ischemia, and 24 hours of reperfusion, using or not different patterns of hypothermia. They were divided in Control group (C): ten animals in normothermic conditions euthanized after anesthesia and stabilization of the homeostasis parameters, without the surgical procedure; Sham group (S): ten animals in normothermic conditions euthanized after simulation of the surgical procedure, with the introduction of the obstructer, but without occlusion of the MCA; Ischemic Control group (IC): 10 animals submitted to 60 minutes of ischemia and 24 hours of reperfusion; Pre-Ischemic Hypothermia group (IH1):10 animals submitted to 30 minutes of pre-ischemic hypothermia extended into the ischemic period itself (plus 60 minutes), and 24 hours of reperfusion; Intra-Ischemic Hypothermia group (IH2): 10 animals submitted to 60 minutes of intra-ischemic hypothermia, returning to normothermia at the beginning of 24 hours of reperfusion; Post-Ischemic Hypothermia group (IH3): 10 animals submitted to 60 minutes of ischemia and 6 hours of post-ischemic hypothermia, initiated at the time of reperfusion. Anesthesia was induced through inhalation of halothane in a glass campanula and kept with a vaporizer linked to a mechanical respirator after endotraqueal intubation. Mean invasive blood pressure was monitored through a PE 50 catheter inserted in the tails' ventral artery, and arterial blood samples were collected after 15 minutes the mechanical ventilation was started and during the final 15 minutes of ischemia, to measure parameters of homeostasis $\left(\mathrm{PaCO}_{2}\right.$, hemoglobin, hematocrit, blood glucose). These parameters were kept according to previous standartization ${ }^{2}$, and blood pressure and $\mathrm{PaCO}_{2}$ were kept adjusting the parameters of the respirator. Body temperature was intermittently recorded using a rectal thermistor and kept between 37 and $38^{\circ} \mathrm{C}$ using a $220 \mathrm{~V}$ lamp next to the animal, for the groups performed using normothermia. Mild $\left(32-34^{\circ} \mathrm{C}\right)$ pre- and intra-ischemic hypothermia were obtained moisten the anesthetized animals with cooled saline solution and reducing the laboratory temperature with air conditioning equipment. Post-ischemic hypothermia was obtained by placing the animals in a container containing ice, immediately after extubation. The animals were kept sedated with gauze immersed in halothane placed on the nose every hour, until the end of six hours. The surgery was performed, according previously described technique ${ }^{16}$. The left cervical vessels were exposed through a ventral midline incision, under the surgical microscope. Obstruction of the MCA was performed by inserting a 4-0 nylon suture (25 $\mathrm{mm}$ long, with a $5 \mathrm{~mm}$ long silicon thickness of $0.28 \mathrm{~mm}$ at one extremity) in the left internal carotid artery, via external carotid artery, until it reach the anterior cerebral artery and occlude the ostium of the MCA. After the reperfusion periods, the animals of the ischemic groups were 
euthanized with massive inhalation of halothane, and underwent thoraco-phreno-laparotomy, the abdominal aorta was clamped and a manual transcardiac perfusion with buffered saline solution ( $0.1 \mathrm{M}$ phosphate buffer, $\mathrm{pH} 7.3$ to $4^{\circ} \mathrm{C}$ ) was performed, followed by infusion of fixative solution of $4 \%$ paraformaldehyde in buffer $0.1 \mathrm{M}$ phosphate at $\mathrm{pH}$ 7.3). The brains were fixed in $10 \%$ buffered formalin, dehydrated cleared and embedded in paraffin. Sequential $10 \mu \mathrm{m}$ thick coronal sections were performed throughout the ischemic area resulting in about a thousand sections for each brain. One in each sequential 15 was selected for coloring, resulting in a total of 60 coronal sections stained for each brain. The sections were stained by Luxol Fast Blue to emphasize the neuronal bodies and non-myelinated fibers in pink-purple, and myelin fibers in blue-green ${ }^{17}$. Digital images of stained sections were captured using analogical-digital camera model JVC TK1270, with 55 mm wide angle lens, set at a vertical holder. A ruler was used for calibration for subsequent conversion of digital measures (pixels) in area measures $\left(\mathrm{mm}^{2}\right)$. The measurement areas of each section were performed using the image analysis program KS400, Carl Zeiss, version 2.0, in a microcomputer IBM-PC and video capture card FG1, Carl Zeiss. The total area of the coronal section, the blue areas (myelin fibers) and red areas (neuron bodies and non-myelinated areas), of both hemispheres were measured successively by the program (Fig 1), and the respective averages of these areas were calculated for each animal. Ratios (percentage) of left (ischemic)/right (normal) hemispheres, for both blue and red areas, were calculated using the average areas ${ }^{18}$. The ischemic area of each section was obtained by the difference between the total area and the sum of all measures in blue and red areas, of both hemispheres. The partial ischemic volume, for 60 stained sections, was calculated of each animal using measurements of ischemic areas, the thickness of each section and the number of sections using Bartustechnique ${ }^{19}$ and corrected when necessary according to Chang technique ${ }^{20}$. The approximate ischemic volume was obtained by the partial volume x 1,000/60. Statistical analysis was performed using the GraphPad InStat program for Windows, version 3.06 (GraphPad Software Inc., San Diego, USA). Analyses of the homeostasis parameters were performed using the analysis of variance (ANOVA) test. For group to group comparison of the percentage of the left hemisphere over the right hemisphere of each animal, of the stained areas, and of the average ischemic areas $\left(\mathrm{mm}^{2}\right)$, and approximated ischemic volumes $\left(\mathrm{mm}^{3}\right)$, was used the nonparametric test of Mann-Whitney-Wilcoxon. A significance level of $\mathrm{p}<0.05$ was considered significant for the two-tailed tests.

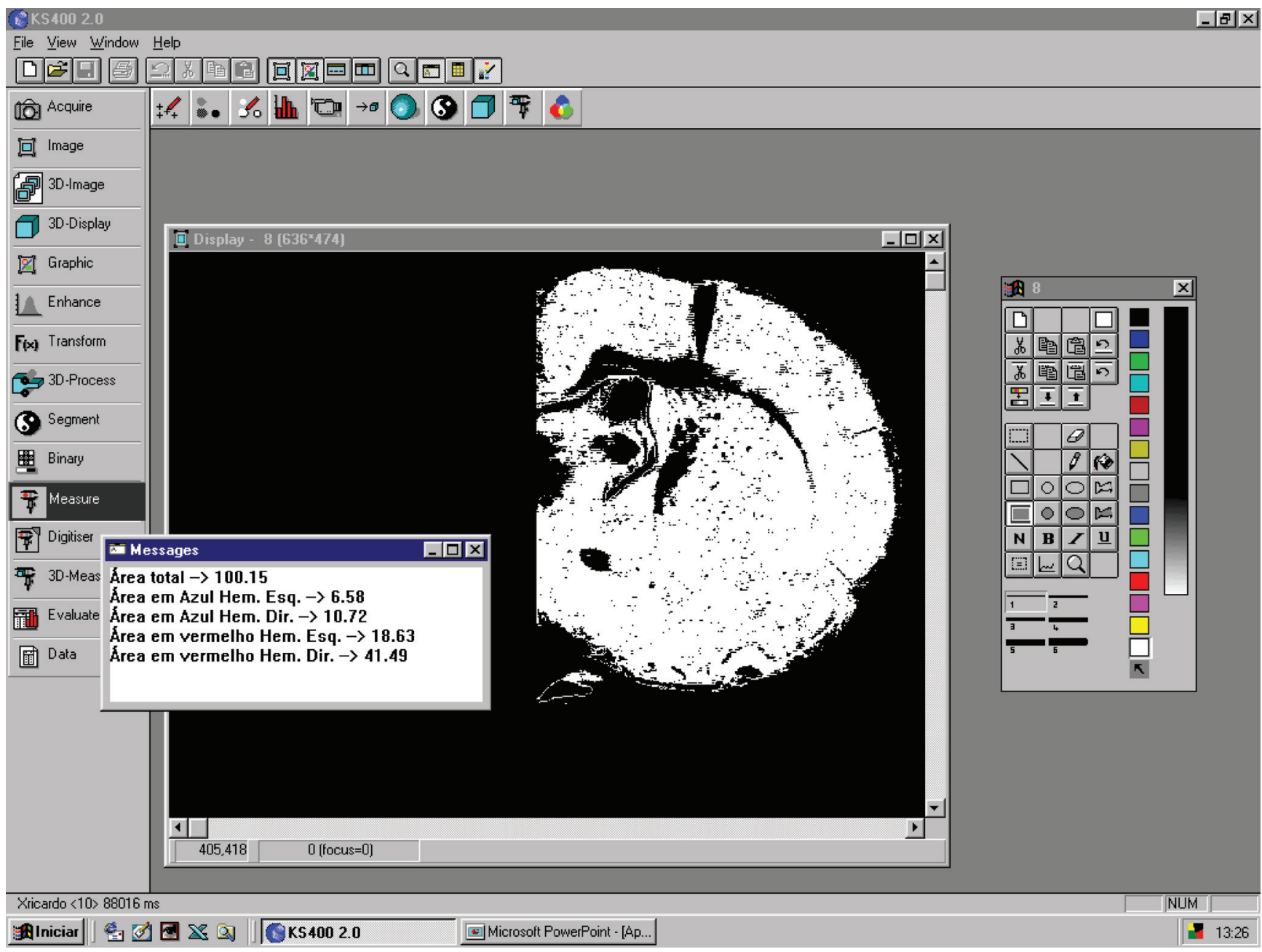

Fig 1. KS400 program work area, with final measurements $\left(\mathrm{mm}^{2}\right)$ of each cut: total area and blue and red areas of both hemispheres. 


\section{RESULTS}

Seventy four male Wistar rats were operated. Of these, six animals were excluded during surgery by inadequate homeostasis parameters. There were eight deaths in this phase ( four animals in IH1 group, two animals in IH3 group, and one animal in IC and IH2 group). All rejected and dead animals were replaced totalizing ten animals per experimental group. The laboratory parameters during surgical procedure were
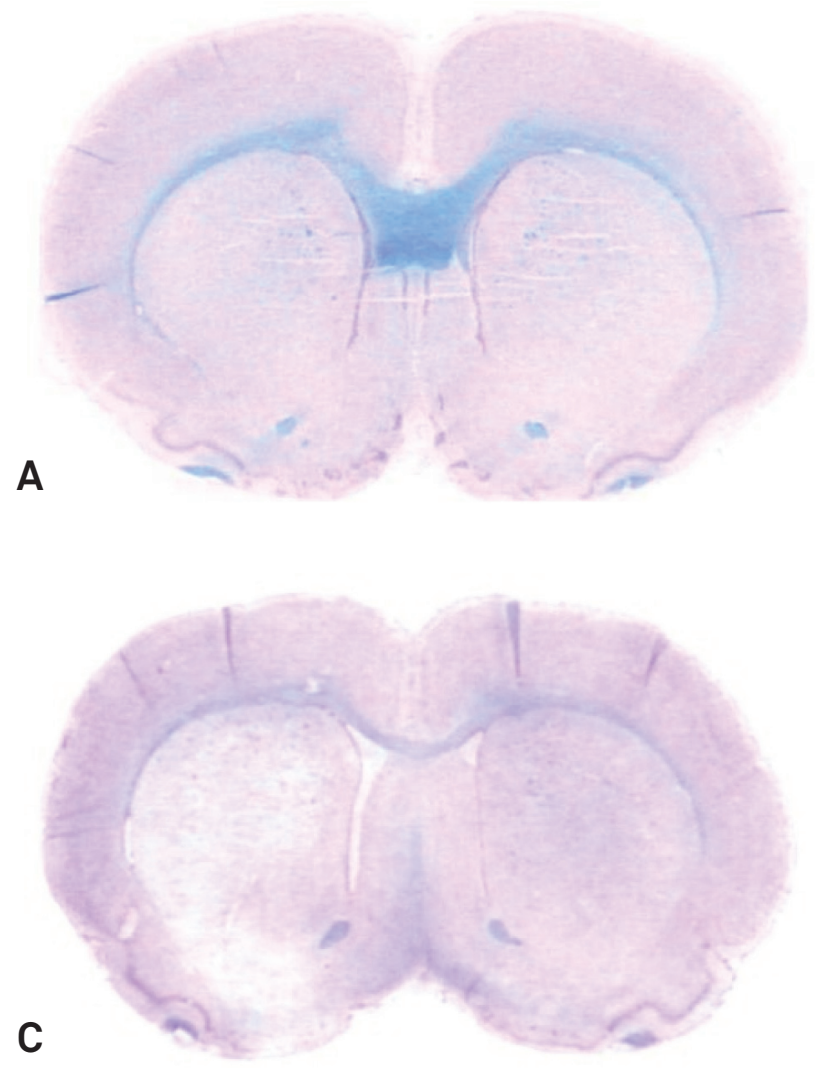

within the limits established for this type of experiment ${ }^{2}$. There was no significant difference among all groups in relation to initial and final values of $\mathrm{PaCO}_{2}$ (respectively, $\mathrm{p}=0.47$ and $\mathrm{p}=0.4$, ANOVA), hemoglobin (respectively, $\mathrm{p}=0.84$ and $\mathrm{p}=0.49$, ANOVA), hematocrit (respectively, $\mathrm{p}=0.57$ and $\mathrm{p}=0.56$, ANOVA), and blood glucose ( $\mathrm{p}=0.34$ and $\mathrm{p}=0.09$, ANOVA). Fig 2 presents coronal sections of brains of animals of control and ischemic groups and Tables 1 and 2 present the average of respectively blue and red areas for the animals of all groups.

Fig 2. Coronal sections of the brain of animals from Control and ischemic groups stained with Luxol Fast Blue (myelinated structures stained in blue-green, and neuronal bodies and non-myelinated structures in pink-purple). (A) Control group. Animals of the Sham group showed similar aspect. (B) Ischemic-control group. Note the left hemisphere ischemic area (clear), extending from basal ganglia to the cortical region. (C) Pre-ischemic hypothermia group. Smaller left hemisphere ischemic area when compared to Ischemic-control group (B). (D) Intra-ischemic hypothermia group. Smaller left hemisphere ischemic area, when compared to Ischemic-control group (B) and similar to Intra-ischemic hypothermia group (C).

Table 1. Averages of blue areas $\left(\mathrm{mm}^{2}\right)$, of both hemispheres, and respective ratios for animals of all experimental groups.

\begin{tabular}{|c|c|c|c|c|c|c|c|c|c|c|c|c|c|c|c|c|c|}
\hline \multicolumn{3}{|c|}{ C } & \multicolumn{3}{|c|}{$S$} & \multicolumn{3}{|c|}{ IC } & \multicolumn{3}{|c|}{ IH1 } & \multicolumn{3}{|c|}{$\mathrm{IH} 2$} & \multicolumn{3}{|c|}{$\mathrm{IH} 3$} \\
\hline $\mathrm{LH}$ & $\mathrm{RH}$ & - $/ \mathrm{RH}$ & LH & $\mathrm{RH}$ & $\mathrm{t} / \mathrm{RH}$ & $\mathrm{LH}$ & $\mathrm{RH}$ & $\mathrm{H} / \mathrm{RH}$ & $\mathrm{LH}$ & $\mathrm{RH}$ & LH/RH & $\mathrm{H}$ & $\mathrm{RH}$ & LH/RH & $\mathrm{LH}$ & $\mathrm{RH}$ & LH/RH \\
\hline 6.31 & 6.69 & 94 & .97 & 7.62 & 091 & 294 & 3 & 0.47 & 4.28 & 39 & 066 & 4.24 & 5.89 & 0.71 & 2.78 & 5.86 & 0.47 \\
\hline 4.7 & & & & & & & & & & & & & & & & & \\
\hline 6.3 & 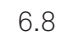 & 93 & 534 & & & & & & & & & 4. & & & & & 47 \\
\hline 4.97 & 5 & 0.87 & 4.79 & 5.25 & 0 & 5. & & & & 6.7 & & 4.4 & & & 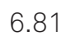 & & 91 \\
\hline 5.96 & $6 \in$ & 0.8 & 5.79 & 6.46 & 0.8 & 4.86 & & & 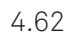 & 7.0 & & 5.3 & & & & & 45 \\
\hline 5.54 & 6 & 0.88 & 5.01 & 5.46 & & 2.7 & 4 & & 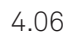 & 6 . & & 4.2 & & . & 2. & & 0.46 \\
\hline 5.9 & 6.38 & 0.92 & 5.25 & 5.64 & 0.93 & 2.37 & 4 & 0. & 3.87 & 5.69 & & 5.5 & 6. & & 6.48 & & 0.94 \\
\hline 5.27 & 5.92 & 0.89 & 5.55 & 6.18 & 0.8 & 2.58 & 5.29 & 0.4 & 4.6 & 6.78 & 0. & 2.3 & 5.2 & 0. & 2.43 & 19 & 0.46 \\
\hline 5.79 & 6.45 & 0.89 & 6.28 & 6.57 & 0.95 & 2.72 & 5.72 & 0.47 & 4.5 & 6.52 & & 3.85 & 5.67 & 0. & 3.37 & 8.28 & 0.4 \\
\hline 5.2 & 5.77 & 0.9 & 5.86 & 6.52 & 0.89 & 2.9 & 6.2 & 0.46 & 4.41 & 6.52 & 0.67 & 5.41 & 5.72 & 0.94 & 3.07 & 6.24 & 0.49 \\
\hline
\end{tabular}

C: Control group; S: Sham group; IC: Ischemic-control group; IH1: Pre-ischemic hypothermia group; IH2: Intra-ischemic hypothermia group; IH3: Post-ischemic hypothermia group; LH: left brain hemisphere; RH: right brain hemisphere.

There was significant difference between C vs. IC, IC vs. IH1, and IC Vs. IH2 groups (respectively, $p=0.0001 ; p=0.01 ;$ and $p=0.03-$ Mann-Whitney-Wilcoxon).There was no significant difference between $\mathrm{C}$ vs. S, IC vs. IH3, and IH1 vs. IH2 groups (respectively, $p=0.39 ; p=0.85 ;$ and $p=0.63-\mathrm{Mann}-\mathrm{Whitney-Wilcoxon)}$ 
Table 2. Averages of red areas $\left(\mathrm{mm}^{2}\right)$, of both hemispheres, and respective ratios for animals of all experimental groups.

\begin{tabular}{|c|c|c|c|c|c|c|c|c|c|c|c|c|c|c|c|c|c|}
\hline \multicolumn{3}{|c|}{ C } & \multicolumn{3}{|c|}{$S$} & \multicolumn{3}{|c|}{ IC } & \multicolumn{3}{|c|}{ IH1 } & \multicolumn{3}{|c|}{$\mathrm{IH} 2$} & \multicolumn{3}{|c|}{ IH3 } \\
\hline $\mathrm{LH}$ & $\mathrm{RH}$ & $\mathrm{LH} / \mathrm{RH}$ & $\mathrm{LH}$ & $\mathrm{RH}$ & $\mathrm{LH} / \mathrm{RH}$ & $\mathrm{LH}$ & $\mathrm{RH}$ & $\mathrm{LH} / \mathrm{RH}$ & LH & $\mathrm{RH}$ & $\mathrm{LH} / \mathrm{RH}$ & LH & $\mathrm{RH}$ & LH/RH & LH & $\mathrm{RH}$ & $\mathrm{LH} / \mathrm{RH}$ \\
\hline 35.38 & 36.42 & 0.97 & 31.9 & 32.9 & 0.96 & 7.25 & 30.51 & 0.23 & 32.01 & 44.7 & 0.71 & 34.43 & 44.52 & 0.77 & 7.51 & 36.35 & 0.2 \\
\hline 34.39 & 35.03 & 0.98 & 35.66 & 36.63 & 0.97 & 8.24 & 34.35 & 0.23 & 34.33 & 35.38 & 0.97 & 34.63 & 45.61 & 0.75 & 35.25 & 36.52 & 0.96 \\
\hline 35.56 & 36.59 & 0.97 & 34.98 & 35.96 & 0.97 & 8.14 & 36.42 & 0.22 & 32.53 & 45.81 & 0.71 & 31.69 & 44.63 & 0.71 & 8.02 & 33.7 & 0.23 \\
\hline 35.04 & 35.91 & 0.97 & 34.22 & 35.9 & 0.95 & 31.85 & 37.04 & 0.85 & 33.93 & 34.96 & 0.97 & 31.78 & 45.43 & 0.69 & 34.82 & 35.85 & 0.97 \\
\hline 34.95 & 35.86 & 0.97 & 35.88 & 37.1 & 0.96 & 30.99 & 35.92 & 0.86 & 25 & 38.19 & 0.65 & 34.42 & 35.75 & 0.96 & 9.16 & 39.54 & 0.23 \\
\hline 34.95 & 36.24 & 0.96 & 35.02 & 36.06 & 0.97 & 7.6 & 37.13 & 0.2 & 31.34 & 44.56 & 0.7 & 31.98 & 44.68 & 0.71 & 8.06 & 39.08 & 0.2 \\
\hline 34.45 & 35.79 & 0.96 & 34.49 & 35.71 & 0.96 & 7.55 & 37.34 & 0.2 & 31.81 & 43.42 & 0.73 & 34.22 & 35.22 & 0.97 & 34.62 & 35.66 & 0.97 \\
\hline 34.82 & 36.05 & 0.96 & 34.4 & 35.59 & 0.96 & 7.22 & 36.24 & 0.19 & 35.06 & 45.66 & 0.76 & 7.08 & 35.82 & 0.19 & 7.24 & 38.04 & 0.19 \\
\hline 35.32 & 36.68 & 0.96 & 35.34 & 36.46 & 0.96 & 7.64 & 35.71 & 0.21 & 32.73 & 45.1 & 0.72 & 31.78 & 43.36 & 0.73 & 8.12 & 31.95 & 0.25 \\
\hline 35.3 & 36.5 & 0.96 & 34.47 & 35.35 & 0.97 & 8.51 & 38.31 & 0.22 & 32.93 & 46.22 & 0.71 & 35.2 & 36.1 & 0.97 & 7.44 & 30.8 & 0.24 \\
\hline
\end{tabular}

C: Control group; S: Sham group; IC: Ischemic-control group; IH1: Pre-ischemic hypothermia group; IH2: Intra-ischemic hypothermia group; IH3: Post-ischemic hypothermia group; LH: left brain hemisphere; $\mathrm{RH}$ : right brain hemisphere.

There was significant difference between C vs. IC, IC vs. IH1, and IC vs. IH2 groups (respectively, $p=0.0001 ; p=0.009 ;$ and $p=0.03-$ Mann-Whitney-Wilcoxon).

There was no significant difference between C vs. S, IC vs. IH3, and IH1 vs. IH2 groups (respectively, $p=0.48 ; p=0.27 ;$ and $p=0.68-M a n n-W h i t n e y-W i l c o x o n)$.

Table 3. Average ischemic areas $\left(\mathrm{mm}^{2}\right)$ for animals of the ischemic groups.

\begin{tabular}{cccc} 
IC & IH1 & IH2 & IH3 \\
\hline 23.36 & 9.07 & 8.23 & 38.23 \\
33.8 & 7.18 & 4.65 & 3.73 \\
35.8 & 7.43 & 8.93 & 32.21 \\
9.51 & 5.58 & 8.45 & 3.13 \\
11.7 & 13.74 & 6.4 & 38.73 \\
37.71 & 9.25 & 9.19 & 38.64 \\
41.72 & 10.43 & 4.81 & 4.98 \\
40.12 & 4.31 & 39.53 & 43.05 \\
37.53 & 9.13 & 10.55 & 24.95 \\
38.14 & 6.43 & 4.75 & 22.7 \\
\hline
\end{tabular}

IC: Ischemic-control group; IH1: Pre-ischemic hypothermia group; IH2: Intraischemic hypothermia group; IH3: Post-ischemic hypothermia group; LH: left brain hemisphere; $\mathrm{RH}$ : right brain hemisphere.

There was significant difference between IC vs. IH1 and IC vs. IH2 groups (respectively, $\mathrm{p}=0.0001$ and $\mathrm{p}=0.0011$ - Mann-Whitney-Wilcoxon). There was no significant difference between $\mathrm{IC}$ vs. $\mathrm{IH} 3$ and $\mathrm{IH} 1$ vs. $\mathrm{IH} 2$ groups (respectively, $p=0.57$ and $p=0.79-$ Mann-Whitney-Wilcoxon).

Concerning ratios of averages of blue areas (myelinated fibers) there was significant difference between groups $\mathrm{C} v \boldsymbol{s}$. IC, IC vs. IH1 and IC vs. IH2 (respectively, $\mathrm{p}=0.0001, \mathrm{p}=0.01$, and $\mathrm{p}=0.03$, Mann-Whitney-Wilcoxon), and there was no significant difference between groups $\mathrm{C} v \boldsymbol{s}$. S, IC $v s$. IH3, and IH1 vs. IH2 (respectively, $\mathrm{p}=0.39, \mathrm{p}=0.85$, and $\mathrm{p}=0.63$, MannWhitney-Wilcoxon). Concerning ratios of averages of red areas (neuronal bodies and non-myelinated fibers), there was significant difference between groups $\mathrm{C} \nu \mathrm{s}$. IC, IC $\nu s$. IH1, and IC vs. IH2 (respectively, $\mathrm{p}=0.0001, \mathrm{p}=0.009$, and $\mathrm{p}=0.03$, MannWhitney-Wilcoxon), and there was no significant difference between groups $\mathrm{C} v \boldsymbol{s}$. S, IC vs. IH3, and IH1 vs. IH2 (respectively, $\mathrm{p}=0.48, \mathrm{p}=0.27$, and $\mathrm{p}=0.68$, Mann-Whitney-Wilcoxon). The analysis for average ischemic areas Table 3 and approximate ischemic volumes Table 4 showed significant difference between IC $\nu$ s. IH1 and IC $v s$. IH2 groups (respectively, $\mathrm{p}=0.0001$ and $\mathrm{p}=0.0011$, Mann-Whitney-Wilcoxon), and showed no significant difference between IC vs. IH3 and IH1 vs. IH2 groups ( $\mathrm{p}=0.57$ and $\mathrm{p}=0.79$, Mann-Whitney-Wilcoxon). Fig 3 presents the approximate ischemic volumes of the ischemic groups.
Table 4. Approximate ischemic volumes $\left(\mathrm{mm}^{3}\right)$ for animals of the ischemic groups.

\begin{tabular}{cccc}
$\mathrm{Cl}$ & $\mathrm{IH} 1$ & $\mathrm{IH} 2$ & $\mathrm{IH} 3$ \\
\hline 239.7 & 92.48 & 83.81 & 389.81 \\
344.76 & 73.1 & 47.43 & 37.91 \\
361.76 & 75.65 & 90.95 & 328.44 \\
96.9 & 56.78 & 86.19 & 31.79 \\
119.34 & 140.08 & 65.28 & 394.91 \\
384.54 & 94.35 & 93.67 & 394.06 \\
425.51 & 106.25 & 48.96 & 50.66 \\
409.19 & 43.86 & 403.07 & 439.11 \\
382.67 & 92.99 & 107.61 & 254.49 \\
388.96 & 65.45 & 48.45 & 231.54 \\
\hline
\end{tabular}

IC: Ischemic-control group; IH1: Pre-ischemic hypothermia group; IH2: Intraischemic hypothermia group; IH3: Post-ischemic hypothermia group; LH: left brain hemisphere; $\mathrm{RH}$ : right brain hemisphere.

There was significant difference between IC vs. IH1 and IC vs. IH2 groups (respectively, $p=0.0001$ and $p=0.0011$ - Mann-Whitney-Wilcoxon). There was no significant difference between IC vs. IH3 and IH1 vs. IH2 groups (respectively, $p=0.57$ and $p=0.79-$ Mann-Whitney-Wilcoxon).

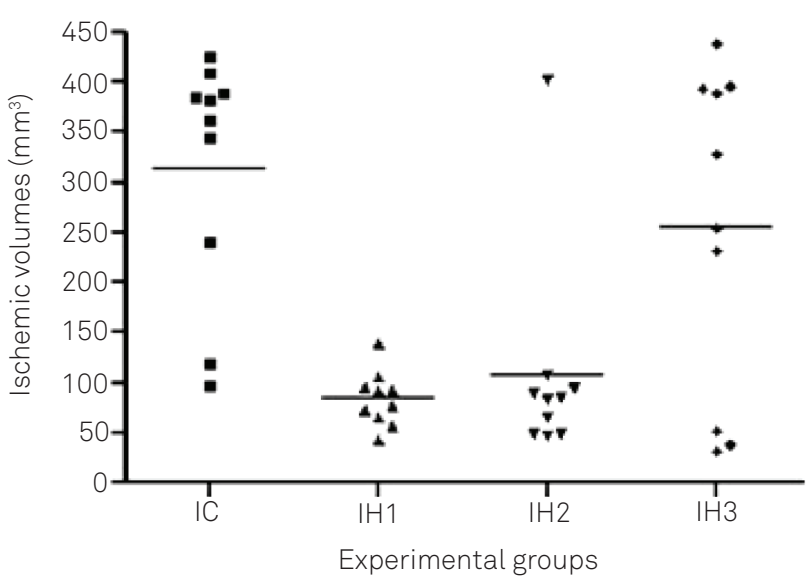

Fig 3. Graphic of approximate ischemic volumes $\left(\mathrm{mm}^{3}\right)$ of the ischemic groups. There was no significant difference between Ischemic-control group (IC) and Post-ischemic hypothermia group (IH3) ( $p=0.57$; Mann-Whitney-Wilcoxon). There was no significant difference between Pre-ischemic hypotermia group $(\mathrm{IH} 1)$ and Intra-ischemic hypotermia group $(\mathrm{IH} 2)(\mathrm{p}=0.79$; Mann-Whitney-Wilcoxon). 


\section{DISCUSSION}

The strain of Wistar rats has the advantage of not suffer a so intense ischemia, and consequently edema, as occurs in spontaneous hypertensive rat (SHR) strain that has little collateral circulation, nor suffer a limited ischemia, as occurs in the Long Evans rat's strain, especially when there is reperfusion, because it has abundant collateral circulation ${ }^{14}$. Male animals were chosen because there is evidence that females have neuroprotection, due to sex specific hormones ${ }^{1}$. Adult animals were used, because younger rats, usually weighing less than $300 \mathrm{~g}$, have more developed collateral vessels, providing greater resistance to infarction ${ }^{21}$. The procedure used to induce ischemia ${ }^{2,16}$ was effective, and also at viable cost. Its great advantage was the possibility of reversion of ischemia, allowing reperfusion when intraluminal obstructer suture is removed, differently from others which use craniotomy with coagulation and/or ligation of the $\mathrm{MCA}^{22}$. This method allows until $70 \%$ of positivity for ischemia, and the fact that some animals do not demonstrate ischemia, in ischemic groups, is possibly due individual anatomical circulation variations of each animal ${ }^{21}$. Deaths occurred mainly in the hypothermic groups, probably due to exposure to low temperature for a long time, and the rate got in this study did not differ from other current studies of the same nature ${ }^{18,23,24}$. The anesthetic agent used for all experimental groups was the halothane, because it does not produce significant changes on intracranial pressure, as well as because of its accessibility and low costeffective ${ }^{25}$. A neuroprotective effect attributed to the halothane is questioned in previous studies ${ }^{18,26}$. In our study this possible effect probably had not an important role because it was used in the same way for all experimental groups. The parameters of the homeostasis $\left(\mathrm{PaCO}_{2}\right.$, hemoglobin, hematocrit and blood glucose) that could somehow change the outcome of ischemic results were always kept within accepted values for this type of experiment ${ }^{2}$, to avoid interference in the ischemic insult. Morphometric analysis of area and volume of focal cerebral ischemia represents an objective way to estimate the extent of ischemic injury and is commonly used for measuring the efficacy of neuroprotective agents ${ }^{7,18,27}$. In this study, we used a new method of semi-automated morphometric analysis using the KS400 computer program, (Carl Zeiss $^{\circledR}$ ), described by Santana ${ }^{18}$. This technique uses coronal sections of the ischemic brain stained with Luxol Fast Blue ${ }^{17}$. This stain allows differentiation between neuronal bodies and myelinated fibers, and the execution of morphometry direct from the lamina, without additional procedures. Moreover, it permits the exclusion of natural empty space, as ventricles and cisterns, or artifactual spaces from histological processing. The method was effective for detecting temporary brain ischemia of 90 minutes, with 30 days $^{18}$. The program provided values for areas stained in blue (myelinated fibers) and red (neuronal bodies), of each hemisphere separately, for each coronal section, and allows to calculate the means for all these values. Because volumes were obtained by multiplying the average ischemic areas by the thickness of the cuts, the average ischemic areas and the approximate ischemic volumes showed similar statistical behavior in the ischemic groups. Hypothermia has been considered the most effective resource, including drugs, to reduce brain injury caused by ischemia in experimental studies ${ }^{25}$. It can change many of the harmful effects consequent to ischemia by suppressing molecular pathways of cell death, as observed in experimental models ${ }^{1}$. Since the first description of the neuroprotective effect of mild hypothermia in intra-ischemic temporary ischemia in rats $^{25}$, numerous experimental studies has demonstrated its beneficial effect. The intra-ischemic modality highly reduces brain infarct size in most experimental model ${ }^{6,7}$. Most studies show that intra-ischemic mild hypothermia in temporary focal ischemia is able to greatly reduce the cerebral infarction ${ }^{14}$. In a meta-analysis of experimental models for the study of cerebral ischemia it was concluded that the intra-ischemic mild hypothermia can reduce infarct volume by $44 \%^{28}$. In rats under the post-ischemic hypothermia, the neuroprotective effect is usually minor ${ }^{10}$, and in experimental studies, there is a window of up to 60 minutes of permanent cerebral ischemia and up to $180 \mathrm{~min}-$ utes for temporary ischemia ${ }^{14,15}$, for what recovery of the ischemic insult can occurs. Increasing the time of the hypothermia may increase its effectiveness ${ }^{14,23}$. The effect of preischemic hypothermia is unclear. In this study, we used mild hypothermia, which is used in most experimental models because fewer side effects ${ }^{28}$, and it was applied before, during and after focal temporary brain ischemia (pre, intra and postischemia). Our results are in agreement with these data. We also noted that neuroprotection occurred for both neuronal bodies and myelinated fibers. Furthermore, we demonstrate for experimental temporary focal ischemia, with 24 hours of reperfusion, that pre-ischemic mild hypothermia, started 30 minutes before ischemia, offers no additional protection compared to the intra-ischemic hypothermia. However, we do not rule out the possibility that pre-ischemic cooling used was insufficient to increase the protection. The neuroprotector effect of post-ischemic hypothermia on brain focal ischemia is controversial. Some authors advocate the use of prolonged hypothermia for 24 hours of reperfusion ${ }^{10}$ or 48 hours ${ }^{11}$ to achieve neuroprotection. However, recent study suggests that it should be initiated in the exact moment of reperfusion and that one hour of duration is enough ${ }^{25}$. Baumann et al. ${ }^{23}$, using mild hypothermia within 6 hours, initiated with reperfusion had a neuroprotective effect, evidenced by preservation of the blood-brain barrier in animals of 6 and 24 hours reperfusion groups. Based on these recent concepts, in the present study we used post-ischemic hypothermia with six hour of duration, started with the reperfusion. However we found no significant difference between animals of the 
post-ischemic hypothermia group and ischemic-control group regarding extension and volume of the ischemia. Although the rectal temperature in animals of experimental groups remained within the limits of mild hypothermia adopted for this study $\left(32-34^{\circ} \mathrm{C}\right)$, it is possible that brain temperature was not at level. This fact is corroborated by a recent study that showed maintenance of rectal temperature at normal levels in animals undergoing temporary cerebral ischemia with cerebral hypothermia induced by infusion of intracerebral cold blood, resulting in decreased brain temperature and rectal temperature remained normal ${ }^{24}$. Therefore, these authors recently advocated the use of endovascular cooling catheters in the studies of cerebral ischemia in rats, instead of local or systemic cooling. Another fact is that the animals of the post-ischemic group, despite the sedation, had shiver after recovery of anesthesia and this could negatively influenced the maintenance of hypothermia. Because of this, it is recommended the use of drugs that inhibit shiver in experimental studies and in future clinical trials using postischemic hypothermia. Extrapolation of its results for humans being in clinical settings is the main difficulty of experimental studies. Despite the positive effect of hypothermia in experimental studies there is lack of multicenter studies with a large number of patients suffering from cerebral ischemia. Positive results involving survivors of cardiac arrest and infants with hypoxic encephalopathy suggest that hypothermic neuroprotection is likely to be promising ${ }^{25}$, and there is already enough scientific evidence that support the beginning of large clinical studies. Despite the negative finding of the intraoperative hypothermia for aneurysm surgery trial $(\text { IHAST })^{29}$, it is possible that such results may be explained by an insufficient cooling time, followed by rapid rewarming, and, in addition, this study has other limitations such as priority to patients in good neurological status (low risk of ischemic injury) and use as a clinical parameter the Glasgow outcome scale (GOS), which fails to adequately evaluate cognitive functions. In addition, further analysis of the results showed that, especially in specific groups (males, and patients with surgery performed between 8 to 14 days after the hemorrhagic ictus), there was improvement in the final neurological outcome 4 . Given the controversy surrounding the issue, beneficial effects of hypothermia, especially in clinical settings, remains to be better investigated.

In conclusion, under the conditions of this study, the preischemic mild hypothermia caused a neuroprotective effect intransient focal cerebral ischemia in rats, similar to intraischemic mild hypothermia, which was not observed with post-ischemic mild hypothermia.

\section{ACKNOWLEDGEMENTS}

To Department Surgery and Anatomy, Ribeirão Preto Medical School of Universidade de São Paulo for supports this research.

\section{References}

1. Mehta SL, Manhas N, Raghubir R. Molecular targets in cerebral ischemia for developing novel therapeutics. Brain Res Rev 2007;54:34-66.

2. Carlotti Jr. CG, Colli BO, Kazuo JY. Avaliação da isquemia cerebral pela respiração mitocôndria. Modelo experimental. Arq Neuropsiquiatr 2001;59:365-371.

3. Rosomoff HL. Hypothermia and cerebral vascular lesions. I. Experimental interruption of middle cerebral artery during hypothermia.J Neurosurg 1956;13:244-255.

4. Choi R, Andres RH, Steinberg GK, Guzman R. Intraoperative hypothermia during vascular neurosurgical procedures. Neurosurg Focus 2009;26:E24.

5. Qin HP, Mei GH, Wei L, Jiang JY. Effect of profound hypothermia on genomics of hippocampus following complete cerebral ischemia in rats. Neurol Res 2008;30:536-541.

6. Maier CM, Sun GH, Kunis D, Yenari MA, Steinberg GK. Delayed induction and long-term effects of mild hypothermia in a focal transient cerebral ischemia: neurological outcome and infarct size.J Neurosurg 2001;94:90-96.

7. Westermaier T, Zausinger S, Baethmann A, Steiger H, SchmidElsaesser S. No additional neuroprotection provided by barbiturateinduced suppression under mild hypothermic conditions in rats subjected to reversible focal ischemia.J Neurosurg 2000;93:835-844.

8. Toyoda T, Suzuki S, Kassel NF, Lee KS. Intraischemic hypothermia attenuates neutrophil infiltration in the rat neocortex after focal ischemia-reperfusion injury. Neurosurgery 1996;39:1200-1205.
Duarte SG. Campos DA, Colli BO. Functional evaluation of temporary focal cerebral ischemia: experimental model. Arq Neuropsiquiatr 2003;61:751-756.

10. Xiong M, Yang Y, Chen GQ,Zhou WH. Post-ischemic hypothermia for $24 \mathrm{~h}$ in P7 rats rescues hippocampal neuron: association with decreased astrocyte activation and inflammatory cytokine expression. Brain Res Bull 2009;79:351-357.

11. Florian B, Vintilescu R, Balseanu AT, et al. Long-term hypothermia reduces infarct volume in aged rats after focal ischemia. NeurosciLett 2008;438:180-185.

12. Zhang R, Chopp M, Chen H, Garcia JH, Zhang ZG. Postischemic (1 hour) hypothermia significantly reduces ischemic cell damage in rats subjected to 2 hours of middle cerebral artery occlusion. Stroke 1993;24:1235-1240.

13. Kuboyama K, Safar P, Radovsky A, Tisherman SA, Stezoski SW, Alexander $\mathrm{H}$. Delay in cooling negates the beneficial effect of mild resuscitative cerebral hypothermia after cardiac arrest in dogs: a prospective, randomized study. Crit Care Med1993;21:1348-1358.

14. Krieger DW, Yenari MA. Therapeutic hypothermia for acute ischemic stroke: what do laboratory studies teach us? Stroke 2004;35:1482-1489.

15. Ohta H, Terao Y, Shintani Y, Kiyota Y. Therapeutic time window of postischemic mild hypothermia and the gene expression associated with the neuroprotection in rat focal cerebral ischemia. Neurosci Res 2007;57:424-433.

16. Koizumi J, Yoshida Y, Nakazaa T, Oneda G. Experimental studies on ischemic brain edema. A new experimental model of cerebral 
embolism in rats in which recirculation can be introduced in the ischemic area. Jpn J Stroke 1986;8:1-8.

17. Klüver $H$, Barrera E. A method for the combined staining of cells and fibers in the nervous system. J Neuropathol Exp Neurol 1953; 12:400-403.

18. Santana RT. Avaliação clínica e morfológica do efeito do tiopental e da clorpromazina na isquemia cerebral focal temporária em ratos. [PhD thesis]. Ribeirão Preto: Universidade de São Paulo; 2008.

19. Bartus RT, Baker KL, Heiser AD, et al. Postischemic administration of AK275, a calpain inhibitor, provides substantial protection against focal ischemic brain damage. J Cereb Blood Flow Metab 1994; 14:537-544.

20. Chang ML, Yang J, Kem S, et al. Nicotinamide and ketamine reduce infarct volume and DNA fragmentation in rats after brain ischemia and reperfusion. Neurosci Lett 2002;322:137-140.

21. Colli BO, Silva MN, Carlotti Jr CG. Isquemia cerebral experimental. In: Silva Jr OC, Zucoloto S, Beer Jr A (ed.). Modelos Experimentais de Pesquisa em Cirurgia. São Paulo: Robe;1998. p. 643-662.

22. Tamura A, Graham DI, McCulloch J, Teasdale GM. Focal cerebral ischemia in the rat:1. Description of technique and early neuropathological consequences following middle cerebral artery occlusion. J Cereb Blood Flow Metab1981;1:53-60.
23. Baumann E, Preston E, Slinn J, Stanimirovic D. Post-ischemic hypothermia attenuates loss of the vascular basement membrane proteins, agrin and SPARC, and the blood-brain barrier disruption after global cerebral ischemia. BrainRes 2009;1269:185-197.

24. Wang F, LuoY, Ling F, et al. Comparison of neuroprotective effects in ischemic rats with different hypothermia procedures. Neurol Res 2010;32:378-383.

25. Ginsberg MD. Neuroprotection for ischemic stroke: past, present and future. Neuropharmacology 2008;55:363-389.

26. Warner DS, McFarlane C, Todd MM, Ludwig P, McAllister AL. Sevoflurane and halothane reduce focal ischemic brain damage in the rat. Possible influence on thermoregulation. Anesthesiol 1993; 79:985-992.

27. Dias LAA, Colli BO, Coutinho Netto J, Lachat JJ. Avaliação da isquemia cerebral focal em ratos. Arq Neuropsiquiatr 2000;58:1047-1054.

28. van der worp hb, Sena ES, Donnan GA, Howells DW, Macleod MR. Hypothermia in animal models of acute ischaemic stroke: a systematic review and meta-analysis. Brain 2007;130:3063-3074.

29. Todd MM, Hindman BJ, Clarke WR, Torner JC, Intraoperative Hypothermia for Aneurysm Surgery Trial (IHAST) Investigators. Mild intraoperative hypothermia during surgery for intracranial aneurysm. N Eng J Med 2005;352:135-145. 